\title{
Delayed Ipsilateral Regional Cortical Injury after Carotid Endarterectomy
}

\author{
Joo Hye Sung \\ Jusun Moon \\ Kyung-Hee Cho \\ Sungwook Yu \\ Department of Neurology, \\ Korea University Anam Hospital, \\ Korea University College of Medicine, \\ Seoul, Korea
}

Received February 18, 2019

Revised April 10, 2019

Accepted April 10, 2019

\section{Correspondence}

Sungwook $\mathrm{Yu}, \mathrm{MD}, \mathrm{PhD}$

Department of Neurology,

Korea University Anam Hospital,

Korea University College of Medicine,

73 Goryeodae-ro, Seongbuk-gu,

Seoul 02841, Korea

Tel +82-2-920-5510

Fax +82-2-926-5347

E-mailssong4yu@gmail.com
Dear Editor,

Cerebral hyperperfusion (or reperfusion) syndrome is a devastating complication of carotid endarterectomy (CEA) in which brain damage, predominantly vasogenic edema, can be identified in imaging modalities soon after the onset of neurologic symptoms. ${ }^{1}$ Here we report on a patient who demonstrated focal neurologic deficits immediately after CEA in the presence of normal initial DWI findings and the delayed appearance of regional cortical injury in the ipsilateral hemisphere in follow-up brain MRI.

A 73-year-old man with diabetes mellitus, hypertension, and scattered cortical infarctions underwent CEA for severe stenosis ( $\geq 70 \%$ ) of the left proximal internal carotid artery (ICA). His vital signs remained stable (systolic blood pressure of 95-145 mm Hg) throughout surgery. An intraluminal shunt was not used intraoperatively because the regional cerebral oxygen saturation measured during intraoperative continuous monitoring of cerebral oximetry with near-infrared spectroscopy was stable after clamping the ipsilateral ICA.

Immediately postoperatively he presented with right-sided weakness and impaired comprehension. DWI performed after symptom onset and on the day after CEA produced unremarkable findings (Supplementary Fig. 1 in the online-only Data Supplement). However, the patient exhibited postoperative confusion and irritability, with the systolic blood pressure fluctuating between 80 and $175 \mathrm{~mm} \mathrm{Hg}$. Electroencephalography performed on the second day after surgery produced normal findings. CT perfusion performed 4 days after CEA showed significantly increased cerebral blood flow in the left hemisphere relative to that preoperatively (Fig. 1A). The blood pressure was maintained between 130/70 and 150/75 mm $\mathrm{Hg}$ from the first postoperative day, while the symptoms of the patient improved thereafter. On the fifth day postoperatively, DWI showed diffuse hyperintense lesions along the left hemispheric cortex on the B1000 map (Fig. 1B), while there were equivocal changes in the signal intensities on the apparent diffusion coefficient and B0 maps in the corresponding area (Supplementary Fig. 2 in the online-only Data Supplement). Brain MRI performed 8 months after left CEA revealed marked atrophy in the left parietotemporal cortex (Fig. 1C). His aphasia further recovered to anomia, but he showed a decline in cognitive function. He scored 15 on the Korean version of the Mini Mental State Examination and 9 out of 30 points on the Korean version of the Montreal Cognitive Assessment.

Delayed cortical injury is an unusual neurologic complication after CEA that probably occurred in the present case after cerebral hyperperfusion or reperfusion. Cortical symptoms occurred a few hours after CEA, while no lesions were found on DWI at least up to 24 hours postoperatively. We found an ipsilateral regional cortical lesion 5 days after the surgery.

The MRI findings in this case are similar to cortical laminar necrosis that is characterized by delayed selective neuronal necrosis of the cerebral cortex and has been reported in hypoglycemic encephalopathy, cerebrovascular diseases, and status epilepticus. ${ }^{2,3}$ To the best of our knowledge, this is the first report of ipsilateral cortical laminar necrosis after CEA. A case

@ This is an Open Access article distributed under the terms of the Creative Commons Attribution Non-Commercial License (https://creativecommons.org/licenses/by-nc/4.0) which permits unrestricted non-commercial use, distribution, and reproduction in any medium, provided the original work is properly cited. 

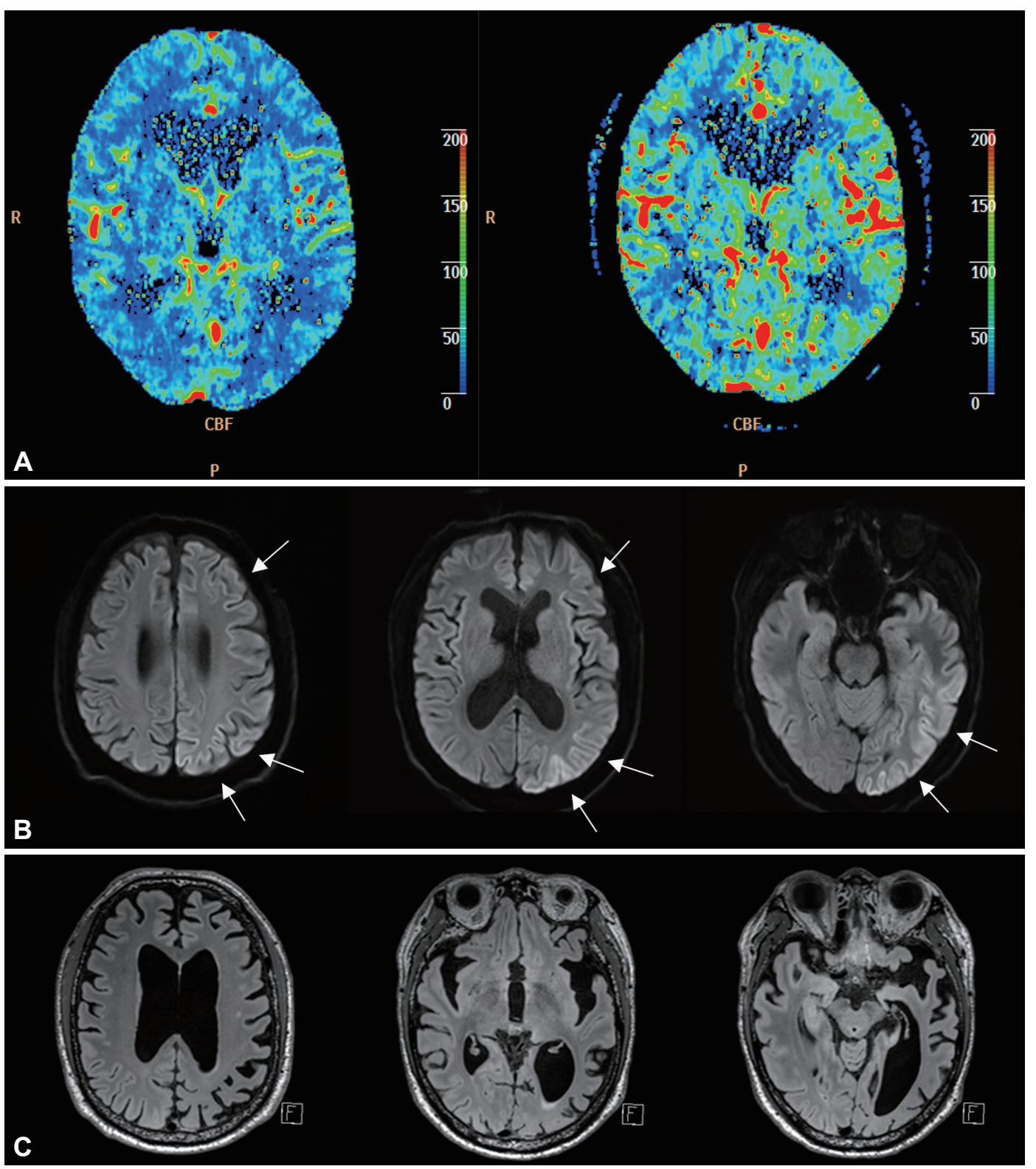

Fig. 1. CTP and brain MRI in a patient with aphasia after left CEA. A: Compared to the preoperative CTP (left panel), CTP on postoperative day 4 (right panel) reveals increased cerebral blood flow in the left hemisphere. B: DWI performed 5 days after left CEA shows diffuse hyperintense lesions along the left hemispheric cortex with a gyral distribution (arrows). C: FLAIR MRI performed 8 months after CEA demonstrates marked atrophy in the left parietotemporal cortex with asymmetric ventricular dilatation. CBF: cerebral blood flow, CEA: carotid endarterectomy, CTP: CT perfusion.

of delayed cortical laminar necrosis associated with hyperperfusion syndrome after extracranial-intracranial bypass surgery for a symptomatic intracavernous ICA aneurysm has been described previously. ${ }^{4}$ In contrast to our case, the lesions in DWI and FLAIR images affected both the cortical and subcortical structures and were already observed on postoperative day 1.

Cognitive impairment occurs in $20-30 \%$ of patients who have undergone CEA. ${ }^{5} \mathrm{~A}$ study using ${ }^{123} \mathrm{I}$-iomazenil singlephoton-emission CT found that postoperative cerebral hyperperfusion was significantly associated with cortical neural loss and cognitive impairment; however, no new lesions were found in serial MRI performed up to 1 month after surgery in patients with postoperative cerebral hyperperfusion. ${ }^{6}$ Our case shows that regional cortical lesions after CEA manifested later as cortical laminar necrosis, which caused encephalomalacic changes.

The ipsilateral regional cortical injury that occurred in the present case may have been related to post-CEA cerebral hyperperfusion. Clamping of the ICA during surgery could induce transient hemispheric ischemia of chronically hypoperfused brain tissue. However, we did not observe any episodes 


\section{REFERENCES}

of hypotension or oxygen desaturation during surgery. The delayed diffuse cortical lesion means that embolism is unlikely to have been the main factor. The pathophysiologic mechanism of delayed cortical injury associated with hyperperfusion/reperfusion after CEA is unclear. The generation of reactive oxygen species with blood reflow might be one mechanism leading to delayed neuronal cell death, ${ }^{7}$ which was partly supported by a case study in which pretreatment with the freeradical scavenger edaravone reduced the incidence of postCEA cognitive impairment. ${ }^{8}$ Progressive cortical neuronal injury might depend on the degree and duration of hyperperfusion/reperfusion after CEA.

Delayed cortical injury is a rare but critical neurologic complication after CEA. Cerebral hyperperfusion or reperfusion could be the mechanism underlying delayed neuronal injury. A better understanding of its pathophysiology would facilitate the appropriate management of neurologic complications after CEA.

\section{Supplementary Materials}

The online-only Data Supplement is available with this article at https://doi.org/10.3988/jcn.2019.15.3.421.

\section{Conflicts of Interest}

The authors have no potential conflicts of interest to disclose.
1. Karapanayiotides T, Meuli R, Devuyst G, Piechowski-Jozwiak B, Dewarrat A, Ruchat P, et al. Postcarotid endarterectomy hyperperfusion or reperfusion syndrome. Stroke 2005;36:21-26.

2. Arbelaez A, Castillo M, Mukherji SK. Diffusion-weighted MR imaging of global cerebral anoxia. AJNR Am J Neuroradiol 1999;20:9991007.

3. Siskas N, Lefkopoulos A, Ioannidis I, Charitandi A, Dimitriadis AS. Cortical laminar necrosis in brain infarcts: serial MRI. Neuroradiology 2003;45:283-288.

4. Iihara K, Okawa M, Hishikawa T, Yamada N, Fukushima K, Iida H, et al. Slowly progressive neuronal death associated with postischemic hyperperfusion in cortical laminar necrosis after high-flow bypass for a carotid intracavernous aneurysm. J Neurosurg 2010;112:1254-1259.

5. Heyer EJ, Sharma R, Rampersad A, Winfree CJ, Mack WJ, Solomon RA, et al. A controlled prospective study of neuropsychological dysfunction following carotid endarterectomy. Arch Neurol 2002;59:217-222.

6. Chida K, Ogasawara K, Suga Y, Saito H, Kobayashi M, Yoshida K, et al. Postoperative cortical neural loss associated with cerebral hyperperfusion and cognitive impairment after carotid endarterectomy: ${ }^{123} \mathrm{I}$-iomazenil SPECT study. Stroke 2009;40:448-453.

7. Traystman RJ, Kirsch JR, Koehler RC. Oxygen radical mechanisms of brain injury following ischemia and reperfusion. J Appl Physiol (1985) 1991;71:1185-1195.

8. Ogasawara K, Yamadate K, Kobayashi M, Endo H, Fukuda T, Yoshida $\mathrm{K}$, et al. Effects of the free radical scavenger, edaravone, on the development of postoperative cognitive impairment in patients undergoing carotid endarterectomy. Surg Neurol 2005;64:309-313. 